\title{
ROBO1 Expression in Metastasizing Breast and Ovarian Cancer: SLIT2-induced Chemotaxis Requires Heparan Sulfates (Heparin)
}

\author{
GÜNTHER A. REZNICZEK ${ }^{1}$, CHRISTINE GRUNWALD ${ }^{1}$, ZIAD HILAL ${ }^{1}$, JAN SCHEICH ${ }^{1}$, \\ GUIDO REIFENBERGER ${ }^{2}$, ANDREA TANNAPFEL ${ }^{3}$ and CLEMENS B. TEMPFER ${ }^{1}$ \\ ${ }^{1}$ Department of Obstetrics and Gynecology, Marien Hospital Herne, Ruhr-Universität Bochum, Bochum, Germany; \\ ${ }^{2}$ Department of Neuropathology, Heinrich Heine University Hospital, Düsseldorf, Germany; \\ ${ }^{3}$ Institute for Pathology, Ruhr-Universität Bochum, Bochum, Germany
}

\begin{abstract}
Background: The members of the slit homolog (SLIT) and roundabout homolog (ROBO) families have emerged as important signaling molecules in tumor metastasis. This study analyzed their role in regulating breast cancer $(B C)$ cell motility and chemotaxis and assessed expression of ROBO1 in brain metastases (BMs) of breast, lung, and colon cancer, and in peritoneal metastases (PMs) of ovarian cancer. Materials and Methods: The BC cell line MDA-MB231 was subjected to scratch, motility, and chemotaxis assays using heparin and a purified recombinant $\mathrm{N}$-terminal SLIT2 fragment. Protein expression was assessed in primary tumors and metastases by immunohistochemistry. Results: Exposure to SLIT2 induced MDA-MB231 cell motility, but no significant chemotaxis without the presence of heparin. ROBO1 was expressed in $4 / 5$ primary $B C$ and in 18/21 BC-derived BM samples; 7/9 BM primary lung cancer samples also stained positive. In contrast, BMs from colorectal cancer were negative for ROBO1. Primary ovarian cancer and ovarian PM showed ROBOl expression in 0/6 and in only 2/6 samples, respectively, whereas SLIT2 was observed in 1/6 primary cancer and in 6/6 PMs samples. Conclusion: SLIT2 can induce BC cell motility and chemotaxis, but the latter requires the presence of heparin. $B M$ expression of ROBO1 is a common feature of some, but not all cancer types. SLIT2 expression appears to be a general feature of ovarian cancer-derived PMs.
\end{abstract}

Correspondence to: Günther A. Rezniczek, Ph.D., Department of Obstetrics and Gynecology, Ruhr-Universität Bochum - Marien Hospital Herne, Düngelstraße 33, 44623 Herne, Germany. Tel: +49 23234991058, Fax: +49 23234991059, e-mail: guenther.rezniczek@ rub.de ORCID: 0000-0002-0852-6002

Key Words: Metastasis, SLIT, ROBO, CXCR4, breast cancer, ovarian cancer, brain metastasis.
Metastasis is a process that involves multiple steps, starting with the mobilization and release of tumor cells from the primary tumor, which are then transported through the lymphatic system and blood vessels to distant sites, where they form new tumors after extravasation and tissue invasion. This process is not random, as it has been established that certain primary cancer types metastasize preferentially to distinct target organs (1). Within the sites of metastasis, distinct molecules, such as cytokines and chemokines play an important role in attracting tumor cells which express the corresponding cell surface receptors (2, 3). C-X-C motif chemokine 12 (CXCL12) with its receptor C-X-C chemokine receptor type 4 (CXCR4), originally identified as a major inducer and regulator of leukocyte chemotaxis (4), is an example of this process. Specifically, CXCR4 is expressed by malignant and pre-malignant breast tumors, such as those at the stage of atypical ductal hyperplasia (5). CXCR4-expressing tumor cells mediate targeted metastasis to bone and other tissues expressing the CXCR4 ligand CXCL12. A new candidate for a modulatory role in CXCR4-/CXCL12-mediated metastasis is the neuronal repellent slit homolog (SLIT) 2 protein. The SLIT proteins (SLIT1, -2, and -3) are a family of large, extracellular-matrix secreted glycoproteins that act as ligands to the Roundabout receptor family (ROBO1, -2, 3 , and -4). They have been linked to neuronal development, myogenesis, leukocyte chemotaxis, and cancer $(6,7)$. SLIT2 (acting through its receptor ROBO1) was found to inhibit CXCL12-induced leukocyte chemotaxis (8). In previous work, we tested whether the SLIT-ROBO crosstalk is active in breast cancer. On a cellular level, we found that SLIT2 indeed induced directed migration of ROBO1-positive breast cancer cell lines (9). Furthermore, subclones of the breast cancer cell line MDA-MB231 with different targets for metastasis exhibited distinct responses to stimulation by CXCL12 and SLIT2. A subclone 
specifically targeting the brain, showed a response to SLIT2, but not to CXCL12, while the situation was reverse for a bone-seeking subclone (9).

The brain is a common site of metastasis in patients with breast cancer, with the reported incidence of clinically symptomatic brain metastases (BM) ranging from $10 \%$ to $15 \%$ (10). Of note, the brain is an organ rich in SLIT2 (11). We found that SLIT2 leads to chemoattraction of subpopulations of breast cancer cells and influences the expression of genes involved in invasion, such as vascular endothelial growth factor (VEGF) and matrix metallopeptidase 9 (MMP9) (9). Thus, we proposed a role for SLIT/ROBO in the metastasis of breast cancer to the brain.

The aims of the present work were to further substantiate the role of SLIT and ROBO in mediating tumor cell metastasis by characterizing SLIT-ROBO signaling in breast cancer cells. In this respect, we specifically wanted to address the role of heparan sulfate proteoglycans, as these play an important role in SLIT-ROBO signaling by forming a ternary complex necessary for signal transduction (12). Furthermore, we analyzed the expression of ROBO1 in BM of patients with breast cancer, as well as other primary cancer types, such as lung and colon cancer. Finally, in an attempt to generalize the role of SLIT-ROBO in tumor cell metastasis, we also looked at primary ovarian cancer and respective metastases to the peritoneum.

\section{Materials and Methods}

Patient samples. Archived tumor samples (primary breast cancer, $\mathrm{n}=5$; $\mathrm{BM}$ of primary breast cancer, $\mathrm{n}=21 ; \mathrm{BM}$ of primary lung cancer, $n=9$; $B M$ of primary colorectal cancer, $n=7$; primary ovarian cancer, $n=6$; peritoneal metastasis of primary ovarian cancer, $n=6$ ) were obtained from our respective pathology archives with Institutional Review Board approval (Ruhr-Universität Bochum, reg. no. 3951-11; and Heinrich Heine Universität Düsseldorf, reg. no. 3511). Informed consent was not necessary.

Cell line. The MDA-MB231 breast cancer cell line was obtained from CLS Cell Lines Service (Eppelheim, Germany; passage number 30). Cells were cultivated in Dulbecco's modified Eagle's minimal essential medium supplemented with $10 \%$ fetal bovine serum, $100 \mathrm{U} / \mathrm{ml}$ penicillin and $100 \mu \mathrm{g} / \mathrm{ml}$ streptomycin (all from Biochrom, Berlin, Germany) and used for experiments between passage numbers 32 to 35 .

Cell migration and motility assays. Purified recombinant human SLIT2-N, a 1,093 amino acid glycoprotein corresponding to the $\mathrm{N}$ terminal portion of the full-length SLIT2 precursor, was obtained from PeproTech (Hamburg, Germany) and used at a concentration of $0.9 \mathrm{ng} / \mathrm{ml}$. Heparin was used at a concentration of $1.5 \mu \mathrm{g} / \mathrm{ml}$. Both were diluted in Dulbecco's modified Eagle's minimal essential medium. Scratch assays were performed by growing cells to confluency in standard cell culture dishes. Perpendicular scratches were made, and fresh medium supplemented with SLIT2-N or vehicle was added. Several scratch intersections were observed for up to $20 \mathrm{~h}$ at regular intervals and the remaining cell-free areas were measured using ImageJ (NIH; https://imagej.nih.gov/ij/).

Cell motility and chemotaxis assays were performed using $\mu$ Slides Chemotaxis 2D (ibidi, Martinsried, Germany) with or without gradient of SLIT2-N with/without heparin applied. $\mu$-Slides provide an elongated, rectangular growth area situated between and open to two opposing reservoir chambers. Thus, after seeding cells, they optionally can be exposed to gradients of soluble components by filling the chambers with corresponding solutions. Cell movement was recorded by videography on a Zeiss Axiovert 25 microscope (Zeiss, Oberkochen, Germany) equipped with an automated stage (13). Phase-contrast images were taken at 10-minute intervals for up to 24 hours. Tracking was performed using a computer program developed in house (sciTaxis) that allows fast and easy semiautomatic tracking of many cells. Using this program, a stack of images is loaded and played as a movie; cells can be tracked manually by following them with the mouse pointer, or by using the built-in auto-tracking mode that follows the center of marked cells with high accuracy. The resulting tracking files (compatible with ImageJ) were then further analyzed using Chemotaxis and Migration Tool 2.0 (ibidi) to determine cell motility parameters such as accumulated distance (total distance traveled by a cell during the observation period), Euclidean distance (linear distance between start and end point), and velocity. For accumulated and Euclidean distances, only cells remaining in the observation area for the whole duration were considered. For velocity, all tracked cells were considered. In experiments where a gradient was applied, the movement of cells parallel to the gradient was considered (forward migration index, FMI). sciTaxis is available for free from one of the authors (GAR) upon request.

Immunohistochemistry. Formalin-fixed, paraffin-embedded tissue samples were processed into 5 - $\mu$ m-thin sections, deparaffinized in xylene and rehydrated in a series of $100-70 \%$ ethanol. Antigen retrieval was performed in sodium citrate solution $(\mathrm{pH} 6,30 \mathrm{~min}$, $100^{\circ} \mathrm{C}$ ). Endogenous peroxidases were quenched with $3 \% \mathrm{H}_{2} \mathrm{O}_{2}$. Slides were blocked with Roti-ImmunoBlock (Carl Roth, Karlsruhe, Germany) and incubated with primary antibodies overnight at $4^{\circ} \mathrm{C}$ : rabbit anti-ROBO1 (ab7279; Abcam, Cambridge, UK), rabbit antiSLIT2 (ab134166; Abcam), mouse anti-CXCR4 (MAB170; R\&D Systems, Wiesbaden, Germany). Peroxidase-conjugated AffiniPure anti-mouse or anti-rabbit antibodies from goat (Jackson ImmunoResearch, West Grove, PA) were applied for $60 \mathrm{~min}$ at room temperature. Color reactions were performed using 3,3'-diaminobenzidine substrate kit and optionally Haematoxylin QS for counterstaining (both from Vector Laboratories, Burlingame, CA, USA). Dehydrated slides were mounted in Mountex (Medite, Burgdorf, Germany) and viewed under an Axioskop2 mot plus (Zeiss). Images were recorded using AxioCam HRc and AxioVision 3.1 software (Zeiss). Samples were classified for expression levels of CXCR4, SLIT2, and ROBO1 by two independent viewers ( $0=$ negative, $1-3=$ weakly, moderately, strongly positive; with increments of 0.5 ).

Statistics. Descriptive data are reported using means and standard deviation for normally distributed data and median and interquartile range (IQR) for data not meeting this assumption. Accordingly, statistical analysis was performed using parametric ( $t$-test) or nonparametric tests (Mann-Whitney $U$-test). Multiple conditions were compared using ANOVA with Bonferroni $t$-test. All $p$-values 
are two-tailed (unless otherwise noted) and $p<0.05$ was considered statistically significant. The statistics software package SigmaPlot 12.5 (Systat Software Inc., San Jose, CA, USA) was used for statistical analysis.

\section{Results}

SLIT2-N increases cell mobility. Exposure of MDA-MB231 breast cancer cells to a purified soluble N-terminal fragment of SLIT2 (SLIT2-N; containing the ROBO1-binding site) led to increased cell motility as demonstrated by scratch assays and tracking of individual cells (all data values: mean \pm standard deviation). The remaining scratch area after $20 \mathrm{~h}$ decreased from $62.3 \pm 12.5 \%$ to $41.1 \pm 9.1 \%$ ( $p=0.038$; one-tailed $t$-test) (Figure 1). When tracking individual cells over $24 \mathrm{~h}$ (without scratch or gradient applied), the accumulated distance travelled by cells $(p=0.003)$, their Euclidean distance $(p=0.003)$, and the velocity of cells $(p<0.001)$ increased significantly (Figure 2$)$. However, when applied in form of a gradient, purified SLIT2-N alone did not induce a detectable chemotactic response (FMI: $-0.96 \pm 0.92$ vs. control $=-0.17 \pm 0.30$ ) (Figure 3 ).

\section{Heparin amplifies SLIT2-induced chemotaxis of breast cancer} cells. Heparin can mimic heparan-sulfate proteoglycans in the formation of a ternary complex with SLIT2 and ROBO1 (12). As shown in Figure 3, a gradient of heparin alone was found to induce chemotaxis of MDA-MB231 cells (FMI: $-1.97 \pm 0.70$ $v s$. control, $p=0.043$ ), whereas a gradient of SLIT2-N did not (see above). A parallel gradient of SLIT2-N and heparin led to even more pronounced chemotaxis (FMI: $-4.60 \pm 0.91 \mathrm{vs}$. control, $p<0.001$; vs. SLIT2-N alone, $p<0.001 ; v s$. heparin alone, $p=0.01)$. In an anti-parallel gradient of SLIT2-N and heparin, cells slightly moved towards heparin (FMI: $0.61 \pm 0.48)$. These results are consistent with the hypothesis that directed SLIT2-dependent migration (attraction) only occurs in the presence of heparan-sulfate proteoglycans.

BMs of primary breast cancer maintain expression of $\mathrm{ROBOI}$ and CXCR4. Weak to moderate expression of ROBO1 was present in $4 / 5$ primary breast cancer samples and weak to strong expression was detected in 18/21 BM samples. Seven out of nine BMs of primary lung cancer also stained positively for ROBO1. In contrast, BMs from primary colorectal cancer were negative for ROBO1 expression (6/7 tumors; only very faint staining in the seventh) (Figure 4A and Supplementary Figures S1-S3, available at https://www.ebi.ac.uk/biostudies/ studies/S-BSST235). Immunoreactivity for CXCR4 was observed in all primary breast cancer samples and in 19/20 breast BM samples, $8 / 8$ lung BM samples, and in 7/7 colorectal BM samples (Figure 4B and Supplementary Figures S1-S3). Thus, both ROBO1 and CXCR4 expression appear to be a general feature of $\mathrm{BM}$ in breast and lung cancer, whereas

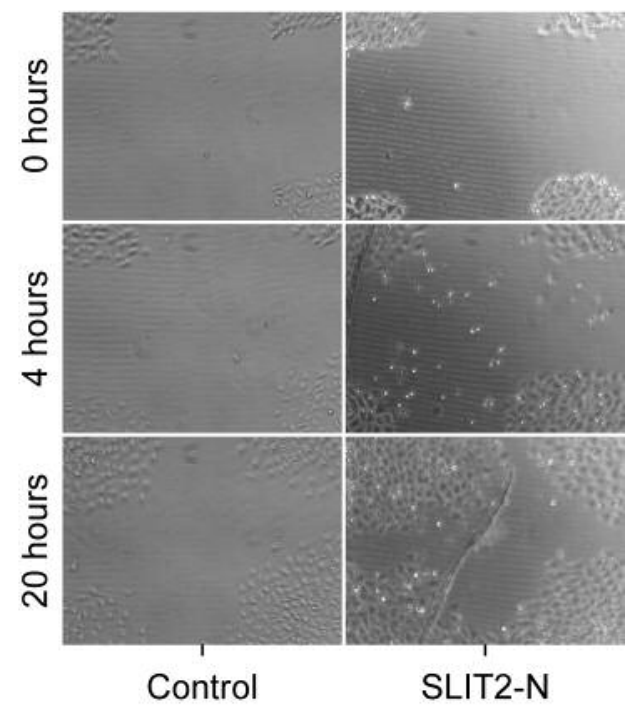

Figure 1. Scratch assay (wound healing). Exemplary phase-contrast micrographs used for quantification of MDA-MB231 cell migration in the absence and presence of $0.9 \mathrm{nM}$ recombinant $N$-terminal slit homolog 2 fragment (SLIT2-N) at the start of the experiment (0 hours) and after 4 and 20 hours.

CXCR4, but not ROBO1, seems to play a role in colorectal BM formation. Exemplary micrographs of ROBO1 and CXCR4 expression in the different sample types are shown in Figure 4C.

Peritoneal metastases (PMs) of primary ovarian cancer upregulate expression of SLIT2 and partially of $\mathrm{ROBO}$. ROBO1 was not expressed in 6/6 primary ovarian cancer samples and weak expression of ROBO1 was detected in 2/6 PM samples. Weak immunoreactivity for SLIT2 was observed in 1/6 primary ovarian cancer samples and in 6/6 PM samples, demonstrating significant up-regulation (Figure 5 and Supplementary Figure S4, available at https:// www.ebi.ac.uk/biostudies/studies/S-BSST235). Thus, ROBO1 is not expressed in ovarian primary cancer, but seems to be acquired in at least some clones metastasizing to the peritoneum. In contrast, SLIT2 expression appears to be a more general feature of ovarian cancer-derived PM.

\section{Discussion}

Axon guidance molecules, SLITs (secreted glycoproteins) and ROBOs (receptors), have recently emerged as players with important roles in tumorigenesis, cancer progression, and metastasis (14). In previous work, we found evidence that the SLIT-ROBO system may play an important role in the metastasis of primary breast cancer to the brain (9). We demonstrated that SLIT2 acts as a potent chemoattractant for 
A

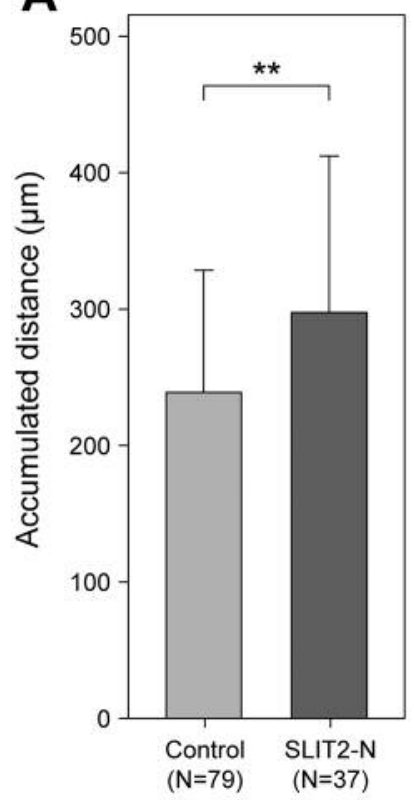

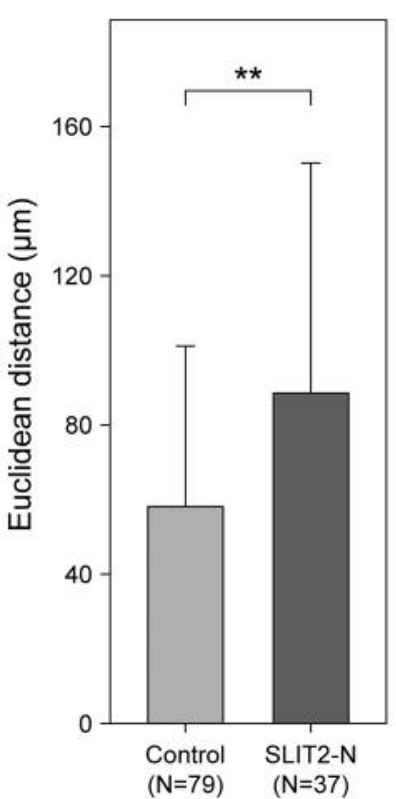

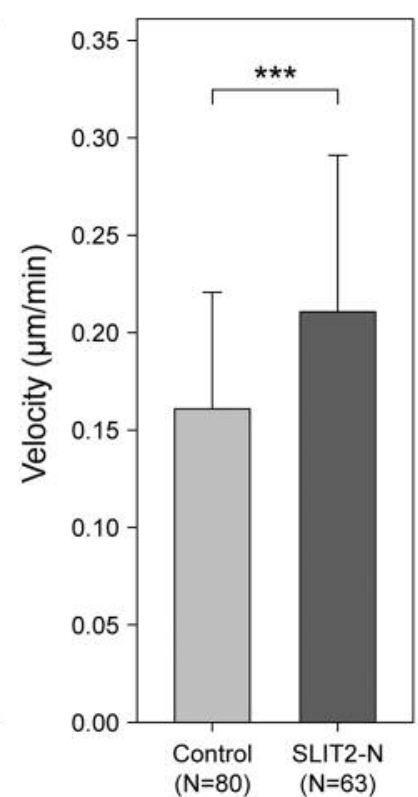

B
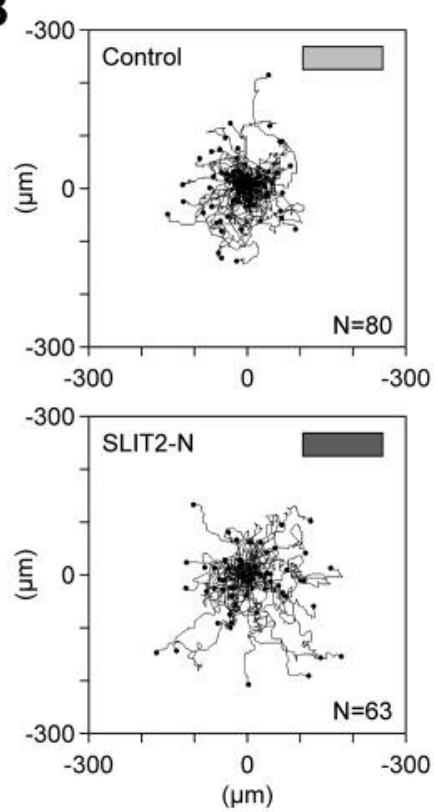

Figure 2. Cell motility assay (videography). MDA-MB231 cells seeded into ibidi $\mu$ Slides were observed for up to 24 h after exchanging the medium with medium containing $0.9 \mathrm{nM}$ recombinant $N$-terminal slit homolog 2 fragment (SLIT2-N) or control medium. A: Bar graphs showing accumulated and Euclidean distances, as well as velocity of tracked MDA-MB231 cells. Data are means \pm standard deviation. B: Tracks of all tracked cells. $N$ : Number of tracked cells (see Materials and Methods for explanation). Significantly different at $* * p<0.01$ and $* * * p<0.001$ (Student t-test).

breast cancer cells and found that cell lines specifically metastasizing to either brain or bone differentially responded to CXCL12 and SLIT2 treatments, suggesting a role for SLIT-ROBO signaling in brain metastasis. In the present study, we further substantiate these findings by characterizing SLIT-ROBO signaling in breast cancer cells and investigating the expression of ROBO1 in BMs of breast and other primary cancer. We specifically addressed the role of heparin, as it plays an important role in SLIT-ROBO signaling by forming a ternary complex necessary for signal transduction (12).

We found that directed SLIT2-dependent migration and attraction only occurred in the presence of heparin. This was clearly demonstrated by the amplified effect of parallel gradients of SLIT2-N and heparin versus SLIT2-N alone. This was in contrast to previous findings where only partially purified SLIT2-N expressed in HEK293 cells was used in a Boyden chamber setup, and thus, some heparan sulfate glycoproteins might have been present (9). The chemotaxisinducing effect of heparin alone is explained by the MDAMB231 cell line expressing considerable levels of SLIT2 (9). We further substantiated these findings on the tissue level by looking at tumor samples of breast, lung, and colorectal cancer, as well as BM from these types of primary cancer. We found both ROBO1 and CXCR4 expression to be a feature of BMs in a subset of breast and lung cancer samples, whereas

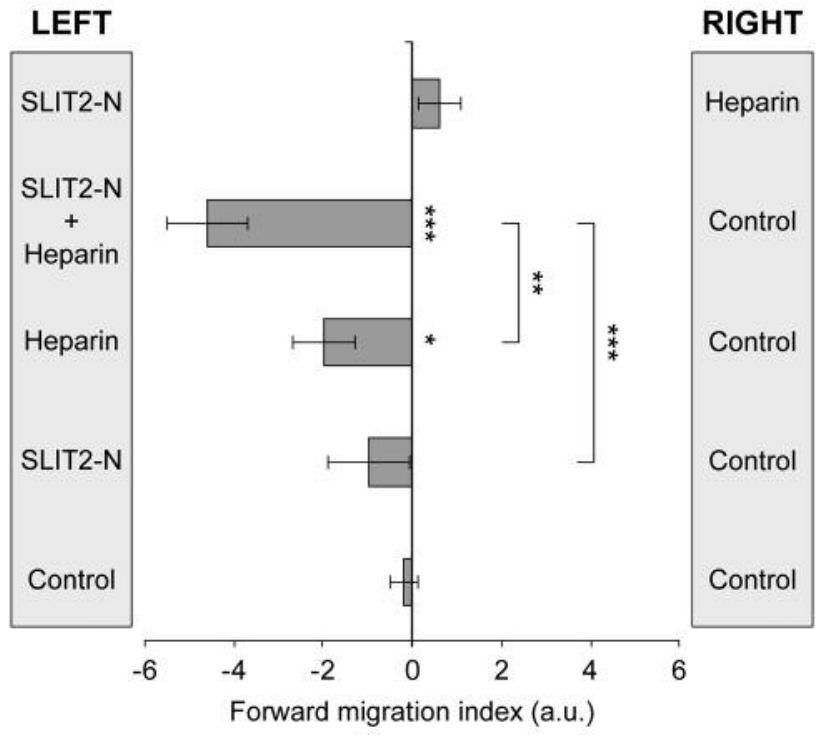

Figure 3. Gradient cell motility assay (videography). MDA-MB231 cells seeded into ibidi $\mu$ Slides were observed for up to 24 h after exposure to the indicated gradients of SLIT2-N with/without heparin. Bar graphs show means of the cell forward migration index (three or more experiments with at least 40 tracked cells per condition). Data are means \pm standard deviation. a.u.: Arbitrary units. Statistics: ANOVA with Bonferroni t-test $(p<0.001)$. Significantly different at $* p<0.5$, $* * p<0.01$, $* * * p<0.001$, or vs. Control/Control. 
A
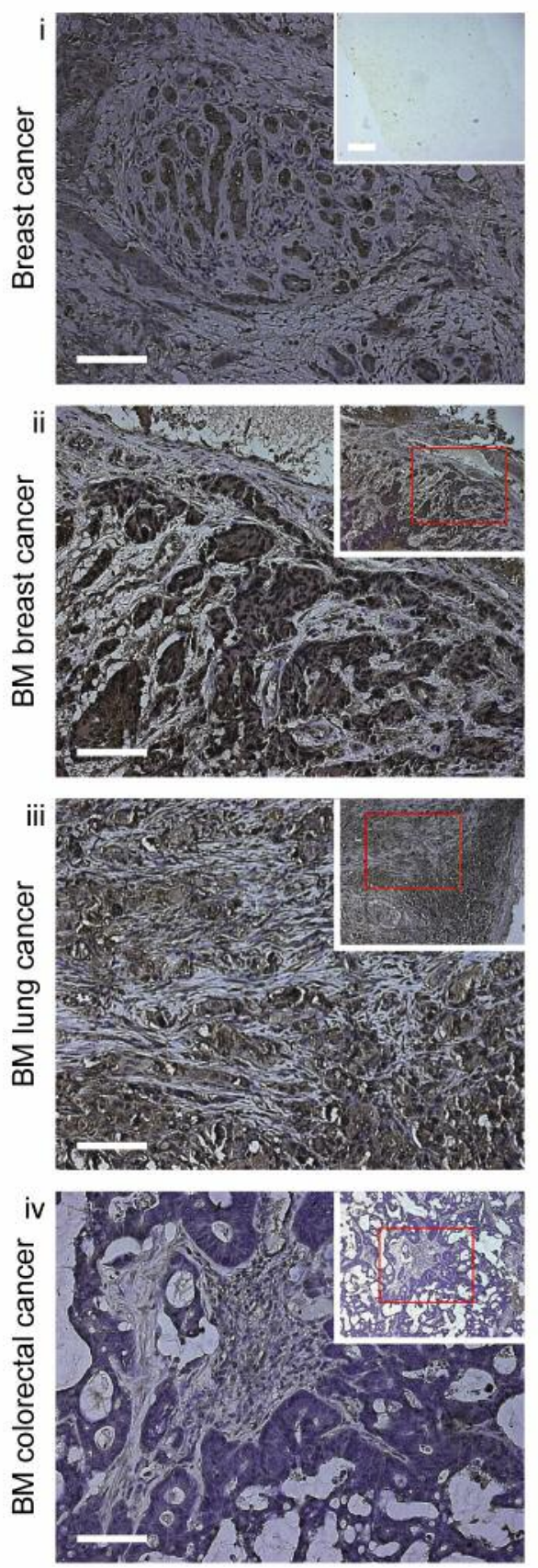

CXCR4
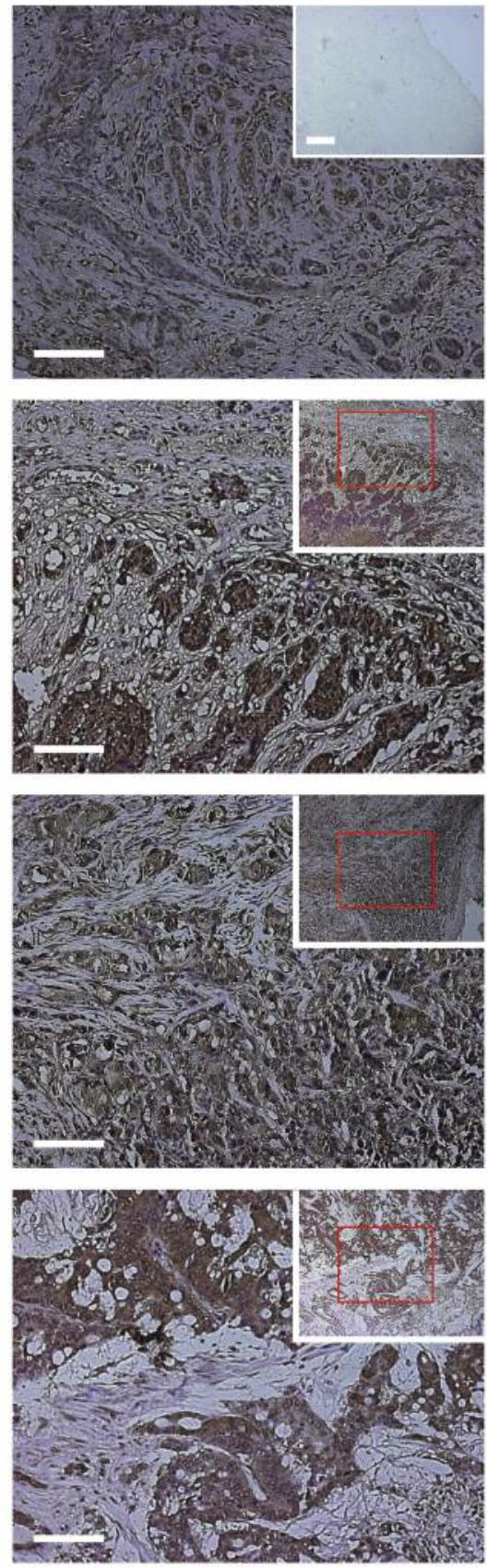

B

ROBO1

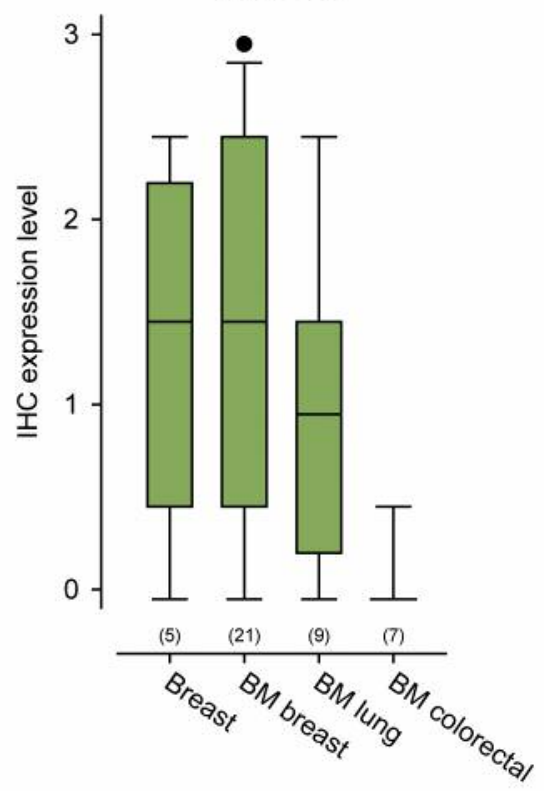

C

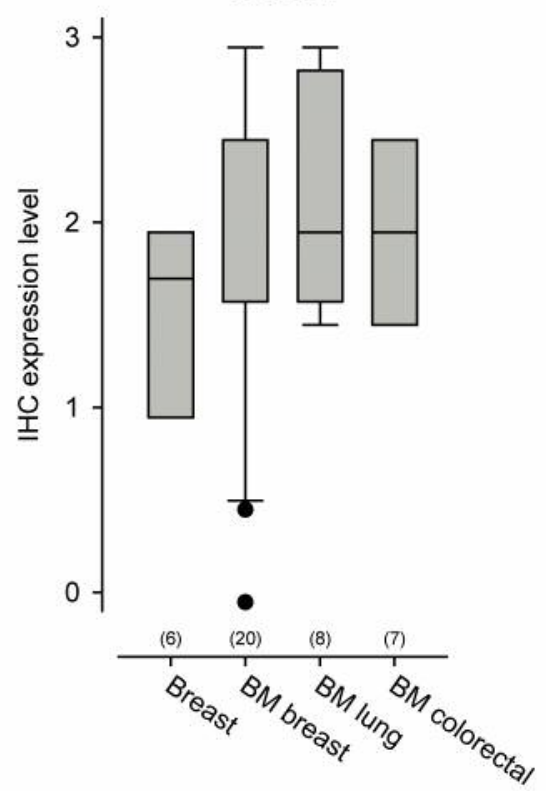

Figure 4. A: Immunohistochemical staining for roundabout homolog 1 (ROBO1) and C-X-C motif chemokine receptor 4 (CXCR4) of primary breast cancer (i) and brain metastases (BM) of breast (ii), lung (iii), and colorectal carcinoma (iv) samples. Exemplary micrographs are shown. Insets in (i) show negative staining controls (no primary antibody; hematoxylin counterstaining omitted). Insets in (ii)-(iv) show lower magnifications (10x); the red frames indicate the area shown in the main panels (20x). Bars=100 $\mu m$. B, C: Box plots for quantification of antibody reactivity for ROBOI (B) and CXCR4 (C). Reactivity was classified as negative (score 0 ) or weakly, moderately, strongly positive (score 1-3). Lower/upper boundaries of boxes represent the 25th/75th percentiles; middle lines the medians; whiskers the 10th and 90th percentiles; and dots are outliers. Numbers in parentheses indicate the number of cases analyzed. See also Supplementary Figures S1-S3 available at https://www.ebi.ac.uk/biostudies/studies/S-BSST235. 

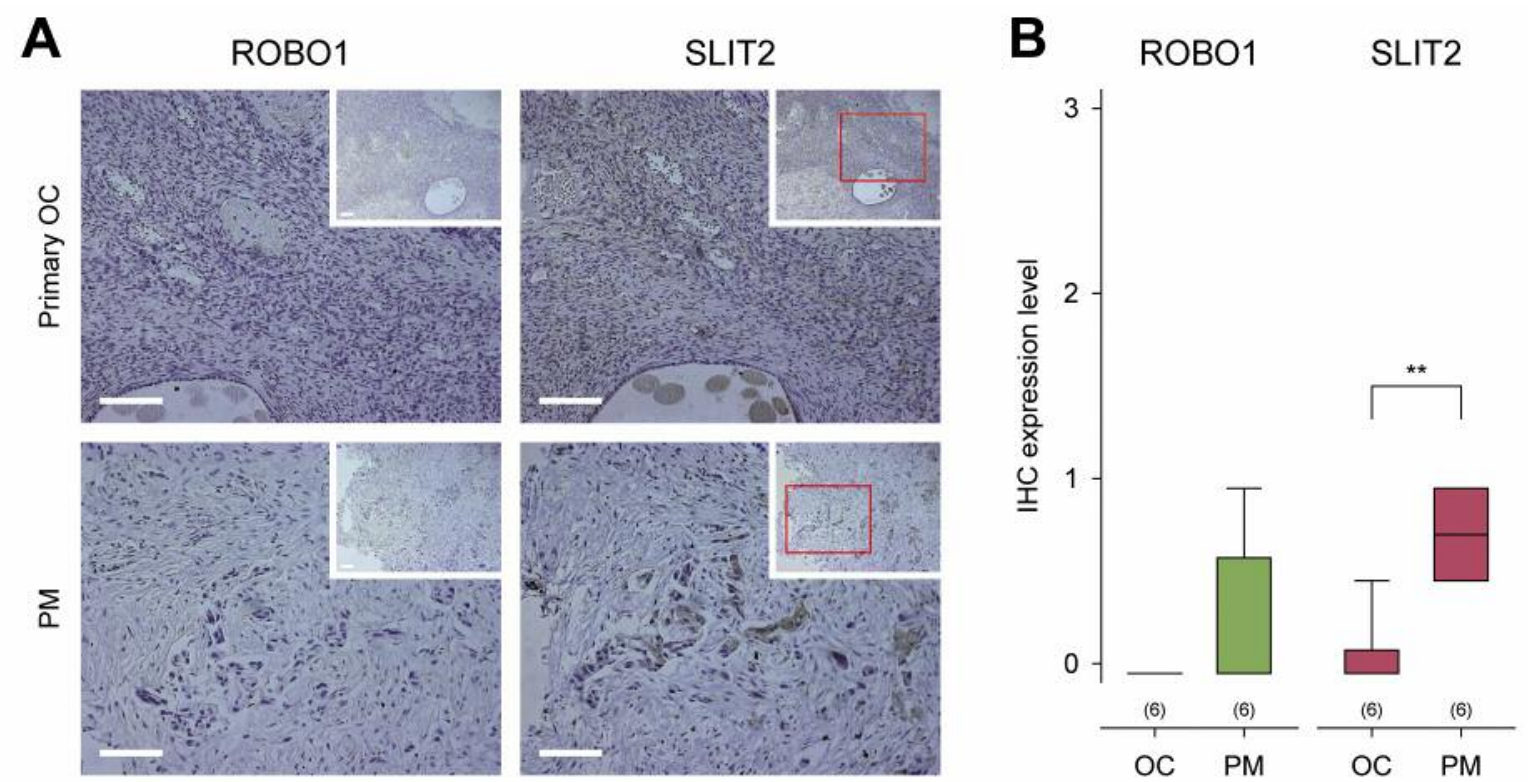

Figure 5. A: Immunohistochemical staining for roundabout homolog 1 (ROBO1) and slit homolog 2 (SLIT2) of primary ovarian cancer (OC) and peritoneal metastases $(P M)$. Exemplary micrographs are shown. Insets in the left column show negative staining controls (no primary antibody). Insets in the right column show tissues at lower magnifications $(10 \times)$; the red frames indicate the area shown in the main panels $(20 \times)$. Bars= $100 \mu \mathrm{m}$. B: Box plots for quantification of antibody reactivity. Antibody reactivity was classified as negative (score 0) or weakly, moderately, strongly positive (score 1-3). Lower/upper boundaries of boxes represent the 25th/75th percentiles; middle lines the medians; whiskers the 10th and 90th percentiles; and dots are outliers. Numbers in parentheses indicate the number of cases analyzed. **Significantly different at p<0.01 (Mann-Whitney U-test). See also Supplementary Figure S4 available at https://www.ebi.ac.uk/biostudies/studies/S-BSST235.

CXCR4, but not ROBO1, seems to play a role in formation of colorectal BM. We further generalized the role of SLITROBO in tumor cell metastasis by demonstrating that ROBO1 is not expressed in primary ovarian cancer, but seems to be acquired in at least some clones metastasizing to the peritoneum. In addition, SLIT2 expression was upregulated in all investigated PMs and thus appears to be a general feature of ovarian cancer-derived PMs.

The role of SLIT-ROBO in ovarian cancer tumorigenesis and tumor cell migration has been discussed controversially. Dickinson et al. assessed SLIT-ROBO expression in benign and malignant ovarian cancer cells (15). They found lower expression in primary cultures of cancer cells compared to normal ovarian epithelium, and poorly differentiated (SKOV3 ) cells compared to well-differentiated (PEO-14) carcinoma cells. Treatment of PEO-14 with cortisol led to reduced expression of SLITs and ROBOs, and lower apoptosis rates were observed in both PEO-14 and SKOV-3 tumor cells after blocking SLIT-ROBO signaling. Increased SLIT-ROBO expression was observed after siRNA-mediated knockdown of the glucocorticoid receptor. These findings indicate that cortisol inhibits SLIT-ROBO expression in ovarian epithelium and in ovarian cancer. Dai et al., on the other hand, investigated the expression of SLIT-ROBO in benign and malignant ovarian tissues and their role in the migration and proliferation of human ovarian cancer cell lines. They identified expression of SLIT2/3 and ROBO1/4 in ovarian cancer cell lines and immunolocalized ROBO1 in many histological types of ovarian cancer. Recombinant human SLIT2, however, did not induce phosphorylation of mitogenactivated protein kinases 1 and 3, or AKT serine/threonine kinase 1, nor lead to a significant increase in tumor cell migration or proliferation. Thus, Dai et al.'s findings suggest that SLIT-ROBO family members are widely expressed by ovarian cancer cells, but the functional role of SLIT-ROBO signaling remains unclear (16).

In conclusion, the findings of our present study argue in favor of a role for SLIT2 and ROBO1 in breast and ovarian cancer. On the cellular level, SLIT2 induced motility and a SLIT2 gradient induced chemotaxis of breast cancer cells, which was amplified by heparin. ROBO1 was abundantly expressed in BMs of breast and colon cancer as well as in PM of ovarian cancer. Together, this suggests that ROBOexpressing tumor cells are attracted to the brain and peritoneum by SLIT2-mediated chemotaxis.

\section{Conflicts of Interest}

No financial or other interests exist with regard to the submitted article that might be construed as a conflict of interest. 


\section{Authors' Contributions}

GAR designed the study, analyzed the data, and wrote the article. CG collected data, performed experiments, and contributed to writing the articIe. ZH analyzed and interpreted data. JS performed experiments. GR and AT provided samples, reviewed the data and critically contributed to the article. CBT assisted in study design and contributed to writing the article. All Authors read and approved the final version of the article.

\section{Acknowledgements}

This work was supported by a grant from the Medical Faculty of the Ruhr-Universität Bochum (FoRUM F711-2011).

\section{References}

1 Rusciano D and Burger MM: Why do cancer cells metastasize into particular organs? Bioessays 14: 185-194, 1992. PMID: 1586372. DOI: $10.1002 /$ bies. 950140309

2 Müller A, Homey B, Soto H, Ge N, Catron D, Buchanan ME, McClanahan T, Murphy E, Yuan W, Wagner SN, Barrera JL, Mohar A, Verastegui E and Zlotnik A: Involvement of chemokine receptors in breast cancer metastasis. Nature 410: 50 56, 2001. PMID: 11242036. DOI: 10.1038/35065016

3 Balkwill $\mathrm{F}$ and Mantovani A: Inflammation and cancer. back to Virchow? Lancet 357: 539-545, 2001. PMID: 11229684. DOI: 10.1016/S0140-6736(00)04046-0

4 Campbell JJ and Butcher EC: Chemokines in tissue-specific and microenvironment-specific lymphocyte homing. Curr Opin Immunol 12: 336-341, 2000. PMID: 10781407. DOI: 10.1016/ S0952-7915(00)00096-0

5 Schmid BC, Rudas M, Rezniczek GA, Leodolter S and Zeillinger R: CXCR4 is expressed in ductal carcinoma in situ of the breast and in atypical ductal hyperplasia. Breast Cancer Res Treat 84: 247-250, 2004. PMID: 15026622. DOI: 10.1023/ B:BREA.0000019962.18922.87

6 Wong K, Park HT, Wu JY and Rao Y: SLIT proteins. molecular guidance cues for cells ranging from neurons to leukocytes. Curr Opin Genet Dev 12: 583-591, 2002. PMID: 12200164. DOI: 10.1016/S0959-437X(02)00343-X

7 Legg JA, Herbert JM, Clissold P and Bicknell R: SLITs and Roundabouts in cancer, tumour angiogenesis and endothelial cell migration. Angiogenesis 11: 13-21, 2008. PMID: 18264786 DOI: $10.1007 / \mathrm{s} 10456-008-9100-\mathrm{x}$
8 Wu JY, Feng L, Park HT, Havlioglu N, Wen L, Tang H, Bacon $\mathrm{KB}$, Jiang Z, Zhang $\mathrm{X}$ and Rao Y: The neuronal repellent SLIT inhibits leukocyte chemotaxis induced by chemotactic factors. Nature 410: 948-952, 2001. PMID: 2072862. DOI: 10.1038/ 35073616

9 Schmid BC, Rezniczek GA, Fabjani G, Yoneda T, Leodolter S and Zeillinger R: The neuronal guidance cue SLIT2 induces targeted migration and may play a role in brain metastasis of breast cancer cells. Breast Cancer Res Treat 106: 333-342, 2007. PMID: 17268810. DOI: 10.1007/s10549-007-9504-0

10 Lin NU, Bellon JR and Winer EP: CNS metastases in breast cancer. J Clin Oncol 22: 3608-3617, 2004. PMID: 15337811. DOI: $10.1200 / \mathrm{JCO} .2004 .01 .175$

11 Qin F, Zhang H, Ma L, Liu X, Dai K, Li W, Gu F, Fu L and Ma Y: Low Expression of SLIT2 and ROBO1 is associated with poor prognosis and brain-specific metastasis of breast cancer patients. Sci Rep 5: 14430, 2015. PMID: 26400100. DOI: 10.1038/srep14430

12 Hohenester E: Structural insight into SLIT-ROBO signalling. Biochem Soc Trans 36: 251-256, 2008. PMID: 18363568. DOI: 10.1042/BST0360251

13 Bühler H, Adamietz R, Abeln T, Diaz-Carballo D, Nguemgo Kouam P, Hero T and Adamietz IA: Automated multichamber time-lapse videography for long-term in vivo observation of migrating cells. In Vivo 31: 329-334, 2017. PMID: 28438859. DOI: 10.21873 /invivo.11063

14 Gara RK, Kumari S, Ganju A, Yallapu MM, Jaggi M and Chauhan SC: SLIT-ROBO pathway. A promising therapeutic target for cancer. Drug Discov Today 20: 156-164, 2015. PMID: 25245168. DOI: 10.1016/j.drudis.2014.09.008

15 Dickinson RE, Fegan KS, Ren X, Hillier SG and Duncan WC: Glucocorticoid regulation of SLIT-ROBO tumour suppressor genes in the ovarian surface epithelium and ovarian cancer cells. PLoS One 6: e27792, 2011. PMID: 22132142. DOI: 10.1371/ journal.pone. 0027792

16 Dai CF, Jiang YZ, Li Y, Wang K, Liu PS, Patankar MS and Zheng J: Expression and roles of SLIT-ROBO in human ovarian cancer. Histochem Cell Biol 135: 475-485, 2011. PMID: 21465248. DOI: $10.1007 / \mathrm{s} 00418-011-0806-2$

Received January 28, 2019

Revised February 18, 2019

Accepted February 21, 2019 